\title{
True Umbilical Cord Knot in a Primigravida
}

Raghav Bhargava ${ }^{1^{*}}$, MRCP (UK) and Madhav Bhargava ${ }^{2}$, MBBS

${ }^{1}$ Department of Endocrinology, University College Hospital, 250 Euston Road, London, United Kingdom.

${ }^{2}$ Department of Internal Medicine, Aster Medcity, Kuttisahib Road Cheranelloor, South Chittoor, Kochi, India, 682087

*Corresponding Author: Raghav Bhargava, Department of Endocrinology, University College Hospital, 250 Euston Road, London, United Kingdom.

Received date: January 31, 2021; Accepted date: April 02, 2021; Published date: April 06, 2021

Citation: R Bhargava, M Bhargava. (2021) True Umbilical Cord Knot in a Primigravida. International Journal of Clinical Case Reports and Reviews. 6(5); DOI:10.31579/2690-4861/112

Copyright: () 2021 Raghav Bhargava, This is an open-access article distributed under the terms of the Creative Commons Attribution License, which permits unrestricted use, distribution, and reproduction in any medium, provided the original author and source are credited.

\begin{abstract}
Umbilical cord knots occur in 1-2\% of all deliveries can lead to increase in fetal death and asphyxia. A thorough cord examination is important to discern abnormalities. Although there are not set guidelines for diagnosing and managing umbilical cord knots; increase of reporting with complications will aid in decision making for better future patient management. We describe one such case in a primigravida who was found to have an umbilical cord knot.
\end{abstract}

Key Words- fetal medicine, umbilical cord knot

\section{Case Report}

A 29-year-old primigravid woman was admitted to our hospital in initial labour at 38 weeks of gestation. She did not have any past medical conditions and there were no complications during pregnancy. Apart from folic acid supplementation she was not on any other medications during the course of her pregnancy. Routine ultrasonography at 20 weeks gestation did not reveal any foetal abnormalities. On admission vital signs were within normal range. The mother's capillary blood glucose was within normal range. Obstetric examination showed a foetus in cephalic position and foetal heart rate was regular. A $3.8 \mathrm{~kg}$ male infant was delivered by normal vaginal delivery. Apgar scores were 9 and 10 at 1 and 5 minutes respectively. Other foetal biometric parameters were normal for gestational age.

Examination of the placenta showed a trivascular umbilical cord of $57 \mathrm{~cm}$ (normal $55-61 \mathrm{~cm}$ ), the diameter was thicker at $3 \mathrm{~cm}$ (normal $1-2 \mathrm{~cm}$ ) and a single true knot was also observed (Figure 1).

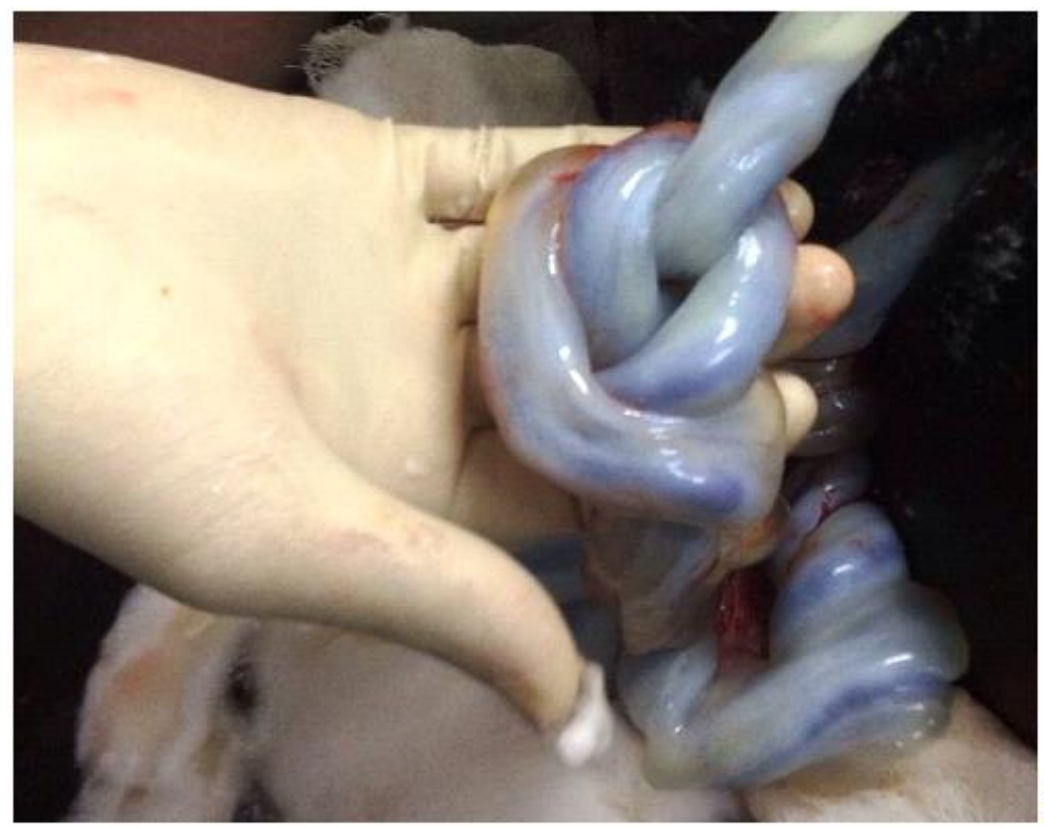




\section{Discussion}

True knots occur in 1-2\% of all deliveries [1]. A true knot is entwining of the umbilical cord segment due to active foetal movements; they are more likely to develop during early pregnancy. Whereas false knots result from kinking of the umbilical vessels leading to localized thickening of Wharton's jelly (gelatinous substance composed of mucopolysaccharides within the umbilical cord).

Risk factors for true knots include gestational diabetes, polyhydramnios, multiparity, long umbilical cord, prolapse of cord and monoamniotic twins [2]. Other umbilical cord abnormalities include loops (coils of long cord around portions of the foetus), torsion and strictures (due to focal deficiency of Wharton's jelly) and haematomas (associated with short cords and entanglement). True knots are easily missed on routine ultrasonography, however four dimensional and colour Doppler examination may help to diagnose them [3].

True umbilical cord knots are unusual to be seen in a primigravida. Associations with true knots include prolonged deceleration of fetal heart rate, meconium stained liquour and fetal death $[4,5]$.

In our case Wharton's jelly was thick (Figure 2) hence it protected the blood vessels of the umbilical cord and prevented a tight knot to form. Although a recurrence of true knots in future pregnancies rare, a history of previous true knots should alert the obstetric team to the need of possible surgical intervention.

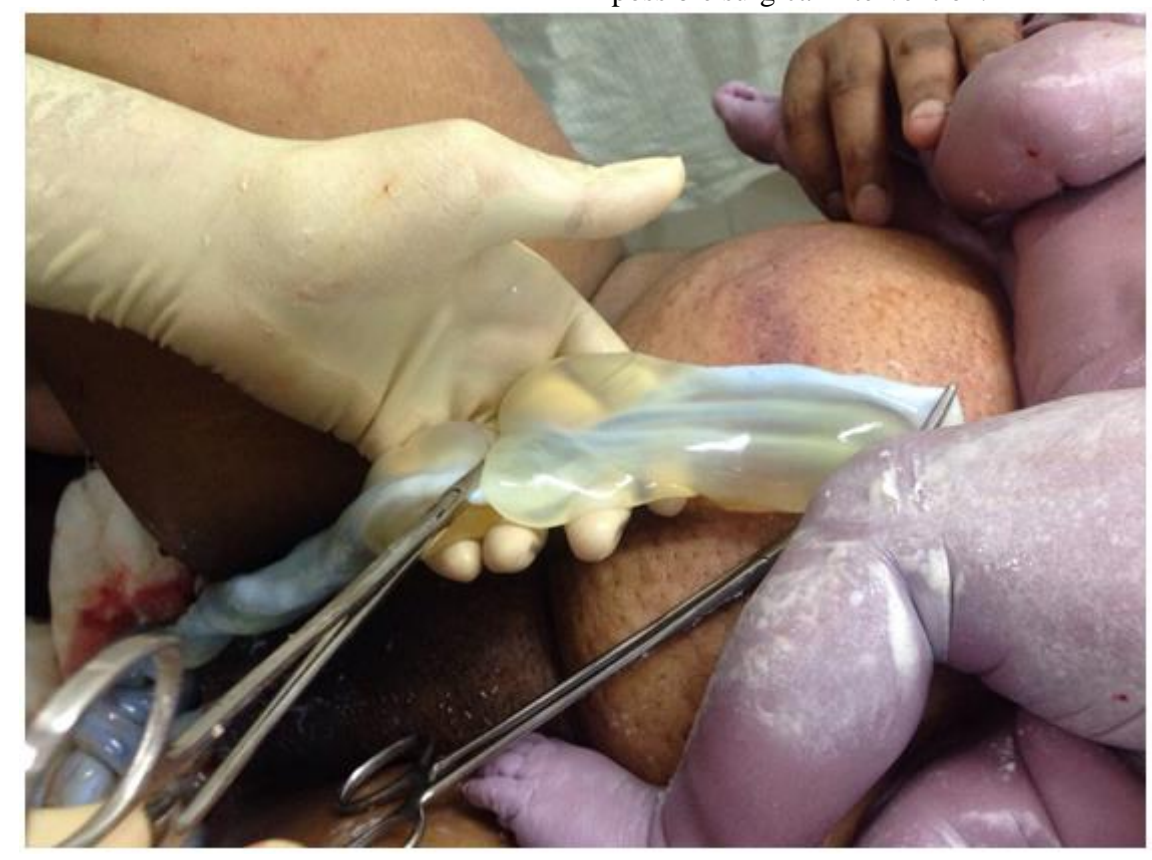

\section{Result}

Both mother and son were discharged from the hospital within 24 hours with no complications.

\section{Contributors}

$\mathrm{RB}$ was involved in writing and editing the manuscript. MB was involved in care of the patient, writing and editing manuscript and image.

\section{Patient Perspective}

The patient was surprised to see an umbilical cord knot as she had not seen one previously and was grateful for all the care provided.

\section{Patient Consent}

Written informed consent gained from patient

\section{References}

1. Spellacy WN, Gravem H, Fisch RO. (1966) The umbilical cord complications of true knots, nuchal coils, and cords around the body. Am J Obstet Gynecol. 94(8):1136-1142.

2. Hershkovitz R, Silberstein T, Sheiner E, Shoham-Vardi I, Holcberg G, Katz M, et al. (2001) Risk factors associated with true knots of the umbilical cord. Eur J Obstet Gynecol Reprod Biol. 98(1):36-39.

3. Guzikowski W, Kowalczyk D, Więcek J. (2014) Diagnosis of true umbilical cord knot. Arch Med Sci. 1:91-95.

4. Srinivasan A, Graves L. (2006) Four True Umbilical Cord Knots. J Obstet Gynaecol Canada. 28(1):32-35.

5. Zbeidy R, Souki FG. (2017) One long umbilical cord, four nuchal cord loops and a true knot. BMJ Case Rep. 223-241. 